\title{
Novel Preparation and Effective Delivery of Mucoadeshive Nanoparticles Containing Anti-diabetic Drug
}

\author{
Sree Harsha Nagaraja ${ }^{1, *}$, Bandar E Al-Dhubiab', Rakesh Kumar Tekade ${ }^{2}$, Katharigatta \\ Narayanaswamy Venugopala ${ }^{1,3}$, Ravindra Vasant Ghorpade ${ }^{4}$, Girish Meravanige ${ }^{5}$, Ahmed Alqadheeb ${ }^{1}$
}

${ }^{1}$ Department of Pharmaceutical Sciences, College of Clinical Pharmacy, King Faisal University, Al-Ahsa, SAUDI ARABIA. ${ }^{2}$ Department of Pharmaceutics, NIPER, Ahmedabad, Gujarat, INDIA.

${ }^{3}$ Department of Biotechnology and Food Technology, Durban University of Technology, Durban, SOUTH AFRICA.

${ }^{4}$ Department of Chemical and Biomolecular Engineering, Yonsei University, Seoul, REPUBLIC OF KOREA.

${ }^{5}$ Department of Biomedical Sciences, College of Medicine, King Faisal University, Al-Ahsa, SAUDI ARABIA.

\begin{abstract}
Background: Vildagliptin is an extensively studied DPP-4 inhibitors (anti-diabetic drug) which has contributed greatly to the understnading of molecular interatcion with the DPP-4 enzyme. However, Vildagliptin exerts glucose- lowering effect transiently due to short elimination half-life of $2-3 \mathrm{hrs}$. Consequently, strict adherence to the dosing schedule strongly emphasised to the diabetic patients. In order to sustain therapeutic effects of a drug at site of absorption, recent efforts have focused on development of mucoadhesive delivery systems using nanoparticles. Methods: Spray-drying method was used to prepare nanoparticles. The drug vildagliptin was incorporated into the nanoparticles and percentage yield, drug content, surface morphology studies, particle size, swelling rate, ex vivo, drug release, drug distribution, histopathology studies were carried out. Results: The yield percentage and drug loading were determined as $95.8 \pm 0.5 \%$ and $69 \pm 0.3 \%$, respectively. SEM microphotographs further revealed nanoparticles with smooth-surfaced spherical forms and particle size were typically about $450 \mathrm{~nm}$ with 375 - 525 range of scattering and swelling rate was found to be $171.5 \pm 12 \%$. The ex vivo studies revealed superior mucoadhesive properties of Vildagliptin nanoparticles $(8.02 \pm 05 \mathrm{hr}$ ). The formulation prolonged the release of Vildagliptin to about $12 \mathrm{hrs}$ (98.5 \pm 2.5$)$. Vildagliptin prolonged retention in GIT $(\sim 12 \mathrm{~h})$ further suggests benefit associated with oral delivery of nanoparticles with higher efficiency than single dose administration. Conclusion: The mucoadhesive nanoparticles optimized in this study may potentially serve as the ideal technology for the effective Vildagliptin delivery specially for diabetes treatment.
\end{abstract}

Key words: Burst release, Diabetes mellitus, Nanoparticle, Muoadhesive, Vildagliptin, Spray drying.

\section{INTRODUCTION}

Diabetes is one of the most alarming public health threats of the current era with a rapidly rising prevalence of 425 million people worldwide projected to reach 629 million by $2045 .{ }^{1}$ The prevalence of diabetes in 2017 for the Middle East and North Africa (MENA) region reached 39 million people and forecasted to double by 2045 to 82 million. ${ }^{1}$ As part of MENA region, the fast- growing population of Saudi Arabia (over 32 million) is not immune to such serious diabetes epidemic with recent estimates indicating more than $25 \%$ of the population affected with diabetes. ${ }^{2}$ Furthermore, in recent years expenses associated with diabetes treatment in Saudi Arabia has surged greater than $500 \%,{ }^{3}$ with the current economic burden estimated to be nearly $14 \%$
Submission Date: 14-10-2018; Revision Date: 05-12-2018; Accepted Date: 22-01-2019

DOI: 10.5530/ijper.53.2s.47 Correspondence: Dr. Sree Harsha Nagaraja, Department of Pharmaceutical Sciences, College of Clinical Pharmacy, King Faisal University, Al-Ahsa, SAUDI ARABIA. Phone: +96-653-548-5322 E-mail: sharsha@kfu.edu.sa

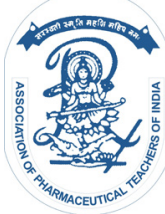

www.ijper.org 
of the total health care expenditure. ${ }^{4}$ According to the International Diabetes Federation data other countries in the Gulf Region with the highest diabetes prevalence include the United Arab Emirates (17.3\%); Bahrain (16.5\%); Qatar (16.5\%), Kuwait (15.8\%); Oman (12.6\%) and Iraq $(8.8 \%){ }^{1}$

Competent management of many patients affected by type 2 diabetes mellitus (T2DM) is restricted due to poor tolerance of some oral antidiabetic drugs during long-term pharmacotherapy. This is compounded by the presence of side effects as well as the inability of the existing drugs to restore secretory capacity of the pancreatic cells. As a result, a surge of interest in newer antidiabetic agents in treatment of T2DM capable of improving islet functions has emerged. ${ }^{5-7}$ The dipeptidyl peptidase-4 (DPP-4) inhibitors are one of the most recent class of anti-diabetic agents with different mechanism of action in management of T2DM that offer clinical benefits to complement activity of wellestablished drugs. ${ }^{8}$

The underlying therapeutic benefit of DPP-4 inhibitors (gliptins) depend on increasing endogenous availability of two incretin hormones mainly glucagon-like peptide-1 (GLP-1) and the gastric inhibitory polypeptide (GIP) by selective inhibitory effect on DPP-4 enzyme which rapidly inactivates these hormones. ${ }^{9,10}$ The incretins in turn bind to their respective receptors promoting secretion of insulin from beta-cells. ${ }^{10}$

Vildagliptin is an extensively studied DPP-4 inhibitors (anti-diabetic drug) which has contributed greatly to the understnading of molecular interatcion with the DPP-4 enzyme. ${ }^{11,12}$ However, Vildagliptin exerts glucose- lowering effect transiently due to short half-life of 2- $3 \mathrm{hrs} .{ }^{13}$ Consequently, strict adherence to the dosing schedule strongly emphasised to the diabetic patients..$^{14,15}$

In order to sustain therapeutic effects of a drug at site of absorption, recent efforts have focused on development of mucoadhesive delivery systems using nanoparticles. ${ }^{16,17}$ Such systems make use of a mixture of the mucoadhesive polymer to form mucoadhesive devices which adhere to mucous intestinal lining releasing drug load slowly. ${ }^{16}$ The mucoadhesive drug delivery systems are advantageous over the other existing delivery systems in that not only they target and localise dosage form specifically, but also result in higher drug flux at the absorption site in the Gastrointestinal tract (GIT), stabilise drug fluctuations in the plasma as well as reduce side effects. ${ }^{18}$

Therefore, this research was designed to evaluate the properties of Vildagliptin release in GIT using a rat model. In addition, a nanoparticle mucoadhesive formulation for Vilagliptin delivery was optimized. Recently albumin used extensively as bioadhesive with controlled release properties to significantly improve delivery of active constituents to various sites has been reported. ${ }^{19}$ On the basis of the published literature so far, this is the first report on delivery system using mucoadhesive drug preparation intended for Vildagliptin-albumin formulation (NVA) and administration.

\section{MATERIALS AND METHODS}

\section{Materials}

Vildagliptin, Albumin was purchased from SigmaAldrich, St. Louis, MO, USA. The remaining chemicals used were of analytical grade. Animals were 6-8 month old male Sprague Dewaley rats purchased from local market Al-Ahsa, Saudi Arabia.

\section{Characterization of formulations}

\section{Preparation of Nanoparticles}

The preparation of albumin-loaded Vildagliptin nanoparticles was performed according to the nano-spray drying method (BUCHI Labortechnik AG, Switzerland) Briefly, a spray nozzle of $4 \mu \mathrm{m}$ was used at $100 \%$ relative fixed spray rate with a $120 \mathrm{~L} / \mathrm{min}$ flow rate. The inlet and outlet temperatures were set at $100^{\circ} \mathrm{C}$, Feed flow rate were between $20 \%$ respectively. The nano-spray drying technique is widely used in pharmaceutical and food science. ${ }^{20}$

A solution of Polymer (1000 mg of Polymer-A soaked in water for 120 mins and pre-warmed before spraying) containing equal amount of Vildagliptin was then filtered to prevent nozzle blockage and spray dried. Subsequently, using a powder scraper the chamber was scraped and dried particles collected to be stored at $25^{\circ} \mathrm{C}$ in a desiccator for later analysis. ${ }^{21}$

\section{Drug content estimation in the formulation}

A know amount of nanoparticles were crushed into fine powder in a mortar and pestle and $100 \mathrm{ml}$ of Phosphate buffered saline (PBS) pH 7.4 and kept for 24 hrs. The solution was stirred for $15 \mathrm{mins}$ and filtrate was analyzed by UV-1601 spectrophotometer (Shimadzu) at a wavelength of $244 \mathrm{~nm}$.

$$
\% \text { Drug content }=\frac{\text { Estimated Drug Content }}{\text { Total Drug Amount Taken }} \times 100
$$

\section{Percentage drug recovery}

The recovery of nanoparticles was measured as the relationship of weight of nanoparticles obtained associated with the total amounts of the drug and polymer at baseline in the preparation process: $:^{22,23}$ 
Nanoparticle recovery $=$ (Amount of nanoparticles collected) / (Total amount of the polymer and drug) $\times 100$

\section{Characterization of Vildagliptin Nanoparticles}

The uncoated Vildagliptin nanoparticles were examined using scanning electron microscopy (SEM) at $20 \mathrm{kV}$ (JSM-6390LA, Joel Ltd, Tokyo, Japan) viewed at 1,000x to $95,000 \mathrm{x}$ range of magnifications. Following platinum sputtering (JFC -1600, Joel Ltd, Tokyo, Japan) various batches were evaluated. ${ }^{22,24}$ To analyze the distribution of the nanoparticles both laser light diffraction and ultrasonic techniques were employed. ${ }^{25}$

\section{Particle size measurement}

Measurement of particle size were carried out using a Malvern Zetasizer (Malvern Zetasizer Nano. ZS, Westborough, MA). The reusable glass cuvette were filled $3 / 4^{\text {th }}$ full with organic solvent and the sample (ultrasonicated), obtained from the spray dryer, were suspended and measured to determine the particle size distribution. ${ }^{26}$

\section{Assessment of Nanoparticle Swelling}

In order to measure the degree of swelling of the nanoparticles, a 1:1 solution of ethanol: methanol was used. Baseline measurements and after incubation with ethanol/methanol solution for $0.15,0.25,0.5,1.0,3.0$, 5.0, 8.0 and 12.0 hrs calculations were performed using microscopy techniques. The proportion of nanoparticle swelling at different time interval was determined by measuring the difference between diameter of nanoparticles at time ' $\mathrm{t}$ ' $(\mathrm{Nt})$ and the initial time $(\mathrm{t}=0[\mathrm{M} 0])$ as follows:

$$
\text { Swelling } \%=\mathrm{Nt}-\mathrm{N} 0 / \mathrm{N} 0 * 100
$$

\section{Characterization of mucoadhesive nanoparticles}

To determine the mucoadhesion characteristics of nanoparticles an in vitro wash-off method described previously was employed. ${ }^{27,28}$ The basis of this experiment involves stimulation of a biological flow to wash test product fixed to a mucous membrane. Briefly, a piece of freshly cut rat stomach mucosa $(7.5 \mathrm{~cm})$ was prepared in isotonic saline solution and secured onto a glass slide with elastic bands. A measured quantity of the nanoparticles was spread over the tissue specimen which was fixed onto a polyethylene support and maintained at $85 \%$ relative humidity for 30 mins in a desiccator. Phosphate buffer solution (PBS) at $\mathrm{pH} 7.4$ was used to wash the mucosa meticulously at a rate of $10 \mathrm{ml} / \mathrm{min}$ using a peristaltic pump. Drug concentration was measured with a spectrophotometer and the quantity of nanoparticles corresponding to the differences in drug levels was calculated. An estimation of adhered nanoparticles was made from calculating the difference between the applied nanoparticles and the flowed nanoparticle amounts. Total proportion of mucoadhesion was then determined from the adhered nanoparticles compared to the actual total of applied nanoparticles.

\section{Drug release study of Vildagliptin}

The patterns of drug release for Vildagliptin was studied in vitro using conventional dialysis method and according to previously described method. ${ }^{29}$ Briefly, in a dialysis bag the Vildagliptin nanoparticles (50 $\mathrm{mg}$ equivalent to nanoparticles) were dialyzed against $250 \mathrm{~mL}$ of Phosphate buffer solution (PBS) which was diluted to a total volume of $1 \mathrm{~L}$ with water $(\mathrm{pH}$ 7.4). The temperature was kept at $37 \pm 1{ }^{\circ} \mathrm{C}$ with the dissolution conditions remaining the same for the remainder of the experiments. $^{29}$

\section{Drug distribution in GIT}

Prior to the experimentation, male Sprague Dawley (SD) rats (250-300 g) were abstained for $20-24 \mathrm{hr}$ period with water provided ad libitum. For the in vivo experiments, a total of five groups each containing 15 rats were used. While the first group was administered aqueous Vildagliptin solution, the rest of the groups were administered Vildagliptin nanoparticles via oral routes. To administer nanoparticles, $50 \mathrm{mg}$ of test sample was suspended in a $\mathrm{ml}$ of saline solution prior to oral administration. After 1, 3, 5, 8 and 12 hrs of oral administration, three rats at each time interval were sacrificed. The stomach tissue (section 1) together with the entire small intestine were isolated and further sectioned into six parts (sections 2-7; length of each section $12 \mathrm{~cm}$ ). ${ }^{30}$ These sections were dissected to expose the inner mucosal surfaces which were scraped to collect all nanoparticles located in each part. The mixture was then pulverised with a homogenizer and Vildagliptin extracted which was then remained at room temperature for 24 hrs. Following centrifugation (805x g) for $20 \mathrm{~min}$, supernatant was analysed spectrophotometrically. This study were conducted according to University Animal Ethical Committee protocol, College of Clinical Pharmacy, King Faisal University, Saudi Arabia approval number (VET/KFU/27/PR-4215).

\section{Histopathological examination of mucosa}

For histopathological examination, after incubation of the collected tissues in PBS (pH 6.4), comparisons were made with the tissues incubated with formulation in the diffusion chamber. Once the collected tissue samples were fixed $(10 \%$ buffered formalin at $\mathrm{pH} 7.2)$, using standard protocols the samples were then paraffin- 
embedded in paraffin. The paraffin sections were then cut at $7.5 \mu \mathrm{m}$ and stained with Hematoxylin and Eosin (HE) on glass slides before being evaluated with light microscopy. This was to identify potential tissue damage during the in vitro process ${ }^{31}$ which was performed by a pathologist blinded to the experiments.

\section{RESULTS AND DISCUSSION}

\section{Drug loading and Percentage Yield}

Spray-drying is a most common interesting techniques for many new drug development studies. The percentage yield and drug loading were determined as $95.8 \pm 0.5 \%$ and $69 \pm 0.3 \%$, respectively. However, low powder yield are reported when prepared in conventional methods. This novel technology can be widely used in different pharmaceutical application due to volume can be as small as $200 \mathrm{mg}^{32}$

The spray-drying method has attracted much attention due to the efficiency of particle generation process and capability for mass production. The nanoparticles of Vildagliptin prepared by this technology exhibited smooth to minor shriveled surfaces (Figure 1). This maybe possibly due to contractions during the drying process in the drying chamber. ${ }^{33}$

\section{Particle Size Analysis}

The Vildagliptin nanoparticles were typically about $450 \mathrm{~nm}$ with 375 - 525 range of scattering (Figure 2). This suggests that the narrow particle size may allow constant release of the Vildagliptin from the nanoparticles at absorption sites. ${ }^{34}$

\section{Swelling Rate}

The swelling rate of Vildagliptin nanoparticles formulation at various time intervals is presented in Figure 3. The outcome revealed that when immersion of the formulation in PBS at $\mathrm{pH} 7.4$ was performed, rapid swelling of all nanoparticles occurred. Previously, swelling has been shown to influence adhesion and cohesion properties of mucoadhesive agents. ${ }^{35}$ As predicted, mucoadhesive nanoparticles tend to use capillary action to withdraw water from the underlying mucosa leading to rapid swelling resulting in a considerably stronger adhesion. ${ }^{30}$ The NVA formulation was found swell by $171.5 \pm 12 \%$.

\section{Mucoadhesion test}

The wash-off studies revealed superior mucoadhesive properties of Vildagliptin nanoparticles $(8.02 \pm 05 \mathrm{hr})$. This adhesive bonding of Vildagliptin nanoparticles to mucin is largely from the existing electrostatic forces and creating hydrogen bonding with mucus molecules resulting in generation of strong adhesive forces. In

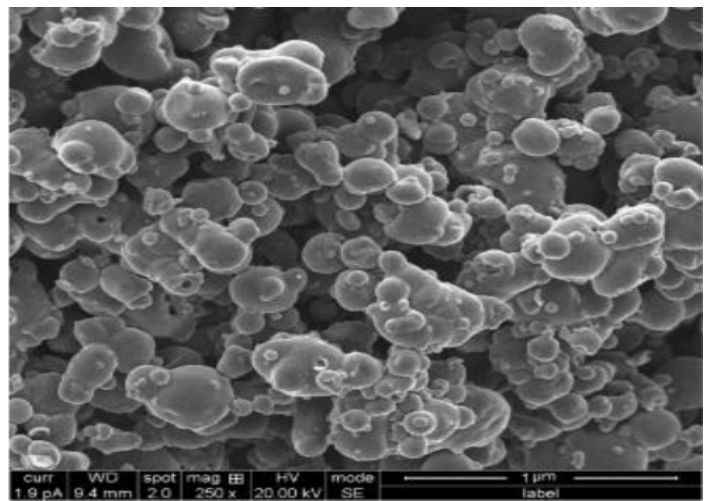

Figure 1: Scanning Electron Microscopy of spray-dried nanoparticles.

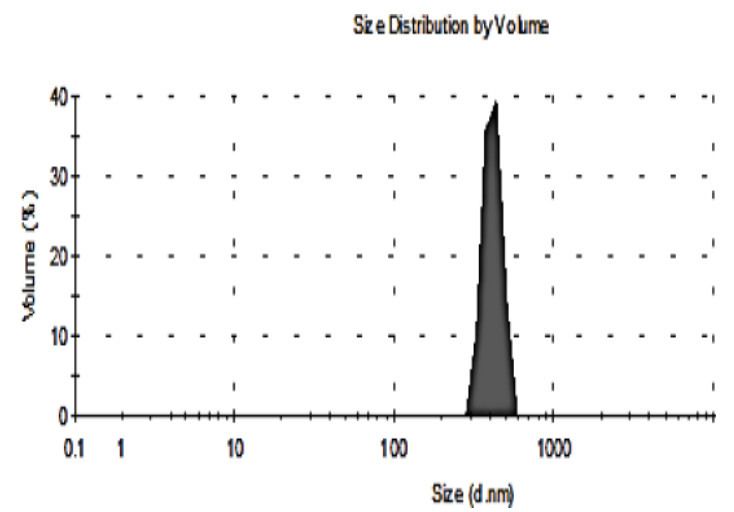

Figure 2: Particle size and distribution of spray-dried nanoparticles $(n=3)$.

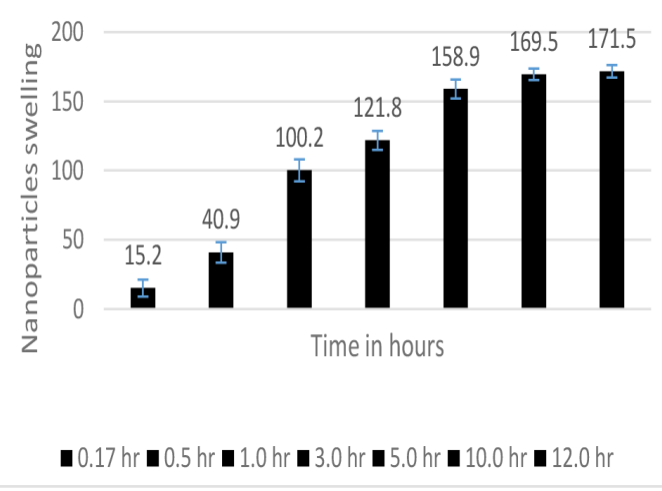

Figure 3: Swelling behavior of nanoparticle formulation $(n=3)$.

turn, drug release into the GIT was made possible due to NVA mucoadhesive properties. In contrast, the control drug was instantly released from the rat gut mucosa supporting lack of mucoadhesive properties in the control drug.

\section{In vitro Release studies}

Nanoparticle formulation exhibits initial rapid release in vitro is depicted in Figure 4. During the first one hour of experimentation, from the total Vildagliptin 


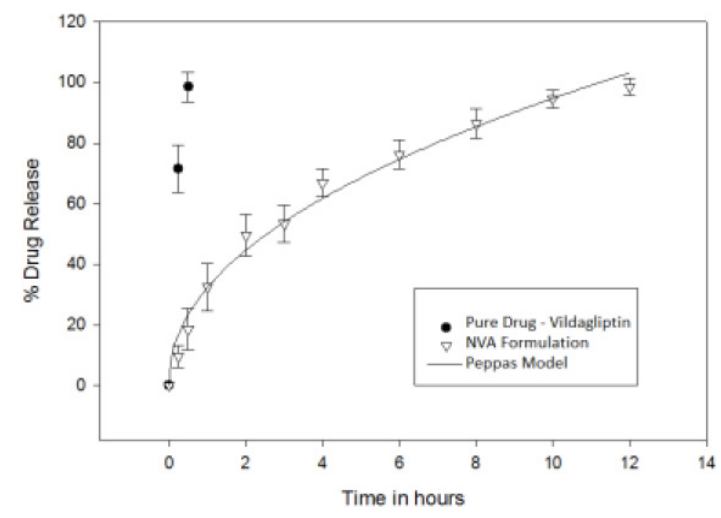

Figure 4: Release kinetics studies of pure vildagliptin and nanoparticles $(n=3)$.

present as part of the NVA nanoparticles almost $32.6 \pm 5 \%$ of the drug was released. This indicated adsorption or weakly fused Vildagliptin on to the large surface of the polymeric nanoparticles. This could be explained as rapid initial burst release followed by prolong release of Vildagliptin to about $12 \mathrm{hrs}$ (98.5 \pm $2.5 \%)$. In contrast, the release of pure drug was rapid (97.3\% released after half an hour). The obtained results from in vitro studies were fitted to different kinetic models (such as Korsmeyer-peppas model, Hixson-Crowell model, Zero order, Higuchi and first-order equations) in order to identify drug release mechanism as well as release rate by applying macros created for the Sigma Plot graphing tool (Systat Software, Trial Version 14.0). ${ }^{29}$ Accordingly, correlation coefficient value $\left(R^{2}\right)$ is taken into account when selection of the most significant curve fit describing it's extend is required. The ideal fit with $R^{2}=0.9751$ was the Korsmeyer-peppas model which was determined by applying various data fits of NVA to identify suitable $R^{2}$ values (Figure 5). The drug release occurs by polymeric matrix diffusion according to the model of Korsmeyerpeppas. The drug release pattern follows the classical Fickian diffusion model whereby nanoparticles initial swelling together with the surface-adsorbed drug particles lead to a 'burst' release display. ${ }^{23}$ Subsequently, owing to diffusion activity, the drug release exhibits a much better control over longer period of time.

\section{In vivo studies}

Figure 5 depicts the percentage of drug concentration, NVA and Vildagliptin administered to Sprague Dewaley rats under experimental conditions as above. The nanoparticles mucoadhesive properties were examined at $30 \mathrm{~min}, 1,3,5,7,10$ and $12 \mathrm{hrs}$

The residual percentage of NVA in the GIT were considerably higher $(P<0.05)$ compared to the Vildagliptin

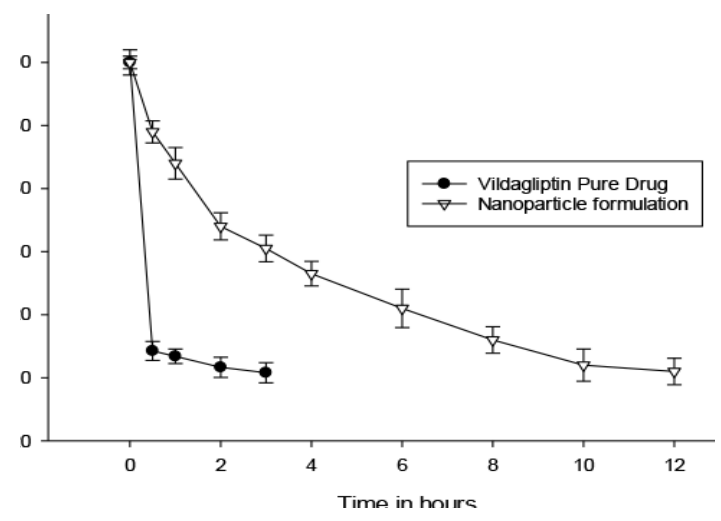

Figure 5: Percentage drug and formulation retained in the stomach $(n=3)$.

suspension. The findings indicated that presence of Albumin in the nanoparticles occurs as a result of their inherent chemical nature. Albumin has no immunogenicity, has half-life of 19 days and has much stronger adhesion under protonated conditions in acidic environments. At time intervals of $30 \mathrm{~min}$ NVA was found to be $-22 \%, 1 \mathrm{~h}-32 \%, 2 \mathrm{~h}-52 \%, 3 \mathrm{~h}-59 \%, 4 \mathrm{~h}-67 \%$, $6 \mathrm{~h}-78 \%, 8 \mathrm{~h}-78 \%, 10 \mathrm{~h}-96 \%$ and at $98 \mathrm{~h}-97 \%$. Compared to the Vildagliptin nanoparticles the drug released at 30 min was $91.5 \%$ with the total drug released $98.4 \%$ at $3 \mathrm{hr}$. At the same time NVA nanoparticles showed $22 \%$ drug release, indicating the great amount of Vildagliptin adsorption or fused near the nanoparticles. This is known as a 'initial rapid release effect' in clinical practice nanoparticles shows faster and effective effect on the patients treatment.

\section{CONCLUSION}

We have developed and characterised an optimized mucoadhesive formulation by evaluation of Vildagliptin release kinetics (in vitro) as well as its distribution in GIT (in vivo) and mucoadhesion properties (ex vivo). The SEM images indicate that nanoparticles were transformed to an almost spherical $375-525 \mathrm{~nm}$ particle size. In addition, the pattern of Vildagliptin release as evident from the in vitro studies follows Korsmeyer-peppas model. At the same time, the nanoparticles exhibit significantly stronger and greater mucoadhesive bonds lasting approximately $12 \mathrm{hrs}$. Vildagliptin prolonged retention in GIT ( $\sim 12 \mathrm{~h}$ ) further suggests benefit associated with oral delivery of nanoparticles with constant drug delivery. We therefore conclude the mucoadhesive nanoparticles in this study may potentially serve as the ideal technology for the effective Vildagliptin delivery specially for diabetes treatment. 


\section{ACKNOWLEDGEMENT}

This study was funding from Deanship of Scientific Research, King Faisal University, Al-Ahsa, Kingdom of Saudi Arabia. (Grant Number: 17122014). Authors thank Mr. Tameem M. Alyahian and Mr. Naif Ali Alunssif (Laboratory Supervisor/ Technician), College of Clinical Pharmacy King Faisal University for logistic support and handling instruments.

\section{CONFLICT OF INTEREST}

The authors declare no conflict of interest.

\section{ABBREVIATIONS}

T2DM: Type 2 Diabetes Mellitus; DPP-4: Dipeptidyl Peptidase-4 (DPP-4); GLP-1: Glucagon-Like Peptide-1; GIT: Gastrointestinal Tract; SD: Sprague Dawley; NVA : Vildagliptin Albumin Nanoparticles.

\section{REFERENCES}

1. D A. International Diabetes Federation. Belgium: International Diabetes Federation. 2017.

2. Alqurashi KA, Aljabri KS, Bokhari SA. Prevalence of diabetes mellitus in a Saudi community. Ann Saudi Med. 2011;31(1):19-23. DOI: 10.4103/02564947.75773

3. Robert AA, AIDawish MA, Braham R, et al. Type 2 Diabetes Mellitus in Saudi Arabia: Major Challenges and Possible Solutions. Curr Diabetes Rev. 2017;13(1):59-64. DOI: 10.2174/1573399812666160126142605

4. Mokdad AH, Tuffaha M, Hanlon M, et al. Cost of diabetes in the Kingdom of Saudi Arabia, 2014. J Diabetes Metab. 2015;6(575):2.

5. Craddy $\mathrm{P}$, Palin $\mathrm{HJ}$, Johnson $\mathrm{KI}$. Comparative effectiveness of dipeptidylpeptidase-4 inhibitors in type 2 diabetes: a systematic review and mixed treatment comparison. Diabetes Therapy. 2014;5(1):1-41.

6. Pan C, Xing X, Han P, et al. Efficacy and tolerability of vildagliptin as add-on therapy to metformin in Chinese patients with type 2 diabetes mellitus. Diabetes, Obesity and Metabolism. 2012;14(8):737-44.

7. Kikuchi M, Haneda M, Koya D, et al. Efficacy and tolerability of vildagliptin as an add-on to glimepiride in Japanese patients with type 2 diabetes mellitus. Diabetes Res Clin Pract. 2010;89(3):216-23.

8. Phung OJ, Scholle JM, Talwar M, et al. Effect of noninsulin antidiabetic drugs added to metformin therapy on glycemic control, weight gain and hypoglycemia in type 2 diabetes. JAMA. 2010;303(14):1410-8.

9. Singh AK. Dipeptidyl peptidase-4 inhibitors: Novel mechanism of actions. Indian J Endocrinol Metab. 2014;18(6):753.

10. Godinho R, Mega C, Teixeira-de-Lemos E, et al. The place of dipeptidyl peptidase-4 inhibitors in type 2 diabetes therapeutics: a "me too" or "the special one" antidiabetic class?. J Diabetes Res. 2015;2015.

11. Chakraborty C, Hsu MJ, Agoramoorthy G. Understanding the molecular dynamics of type-2 diabetes drug target DPP-4 and its interaction with sitagliptin and inhibitor diprotin-A. Cell Biochem Biophys. 2014;70(2):907-22.

12. Mulvihill EE, Drucker DJ. Pharmacology, physiology and mechanisms of action of dipeptidyl peptidase-4 inhibitors. Endocr Rev. 2014;35(6):992-1019.
13. Scheen A. Pharmacokinetics of dipeptidylpeptidase-4 inhibitors. Diabetes, Diabetes Obes Metab. 2010;12(8):648-58.

14. Karagiannis $\mathrm{T}$, Paschos $\mathrm{P}$, Paletas $\mathrm{K}$, et al. Dipeptidyl peptidase-4 inhibitors for treatment of type 2 diabetes mellitus in the clinical setting: systematic review and meta-analysis. BMJ. 2012;344:e1369.

15. Holst JJ, Deacon CF. Inhibition of the activity of dipeptidyl-peptidase IV as a treatment for type 2 diabetes. Diabetes. 1998;47(11):1663-70.

16. Boddupalli BM, Mohammed ZNK, Nath RA, et al. Mucoadhesive drug delivery system: An overview. J Adv Pharm Technol Res. 2010;1(4):381-7. DOI: 10.4103/0110-5558.76436

17. Harsha SN, Aldhubiab BE, Nair AB, et al. Nanoparticle formulation by Büchi B-90 Nano Spray Dryer for oral mucoadhesion. Drug Des Devel Ther. 2015;9:273.

18. Gupta S, Samanta MK, Raichur AM. Dual-drug delivery system based on in situ gel-forming nanosuspension of forskolin to enhance antiglaucoma efficacy. Aaps Pharmscitech. 2010;11(1):322-35.

19. Gilhotra RM, Ikram M, Srivastava S, et al. A clinical perspective on mucoadhesive buccal drug delivery systems. J Biomed Res. 2014;28(2):81.

20. Ré MI. Formulating drug delivery systems by spray drying. Drying Technol. 2006;24:433-46.

21. Harsha S. Dual drug delivery system for targeting $H$. pylori in the stomach: preparation and in vitro characterization of amoxicillin-loaded Carbopol ${ }^{\circledR}$ nanospheres. Int J Nanomedicine. 2012;7:4787.

22. Harsha S, Chandramouli R, Rani S. Ofloxacin targeting to lungs by way of microspheres. Int J Pharm. 2009;380(1-2):127-32.

23. Lu B, Zhang J, Yang $\mathrm{H}$. Lung-targeting microspheres of carboplatin. Int $\mathrm{J}$ Pharm. 2003;265(1-2):1-11.

24. Dyakonov $\mathrm{T}$, Yang $\mathrm{CH}$, Bush D, et al. Design and characterization of a silkfibroin-based drug delivery platform using naproxen as a model drug. J Drug Deliv. 2012;2012.

25. Alexakis T, Boadi D, Quong D, et al. Microencapsulation of DNA within alginate microspheres and crosslinked chitosan membranes for in vivo application. Appl Biochem Biotechnol. 1995;50(1):93-106.

26. Nair AB, Al-Dhubiab BE, Shah J, et al. Poly (lactic acid-co-glycolic acid) Nanospheres Improved the Oral Delivery of Candesartan Cilexetil. Indian J Pharm Educ. 2017;51(4):571-9.

27. Hasçiçek C, Gönül N, Erk N. Mucoadhesive microspheres containing gentamicin sulfate for nasal administration: preparation and in vitro characterization. II Farmaco. 2003;58(1):11-6.

28. Rao KR, Buri P. A novel in situ method to test polymers and coated microparticles for bioadhesion. Int J Pharm. 1989;52(3):265-70.

29. Harsha NS, Rani RS. Drug targeting to lungs by way of microspheres. Arch Pharmacal Res. 2006;29(7):598-604.

30. Dhaliwal S, Jain S, Singh HP, et al. Mucoadhesive microspheres for gastroretentive delivery of acyclovir: in vitro and in vivo evaluation. The AAPS Journal. 2008;10(2):322.

31. Majithiya RJ, Ghosh PK, Umrethia ML, et al. Thermoreversible-mucoadhesive gel for nasal delivery of sumatriptan. AAPS Pharm Sci Tech. 2006;7(3):E80-6.

32. Haggag YA, Faheem AM. Evaluation of nano spray drying as a method for drying and formulation of therapeutic peptides and proteins. Front Pharmacol. 2015;6:140. DOI: 10.3389/fphar.2015.00140

33. Raffin RP, Jornada DS, Ré MI, et al. Sodium pantoprazole-loaded enteric microparticles prepared by spray drying: Effect of the scale of production and process validation. Int J Pharm. 2006;324(1):10-8.

34. Morishita M, Goto T, Peppas NA, et al. Mucosal insulin delivery systems based on complexation polymer hydrogels: effect of particle size on insulin enteral absorption. J Control Release. 2004;97(1):115-24.

35. Mortazavi SA, Smart JD. An investigation into the role of water movement and mucus gel dehydration in mucoadhesion. $J$ Control Release. 1993;25(3):197-203. 
PICTORIAL ABSTRACT

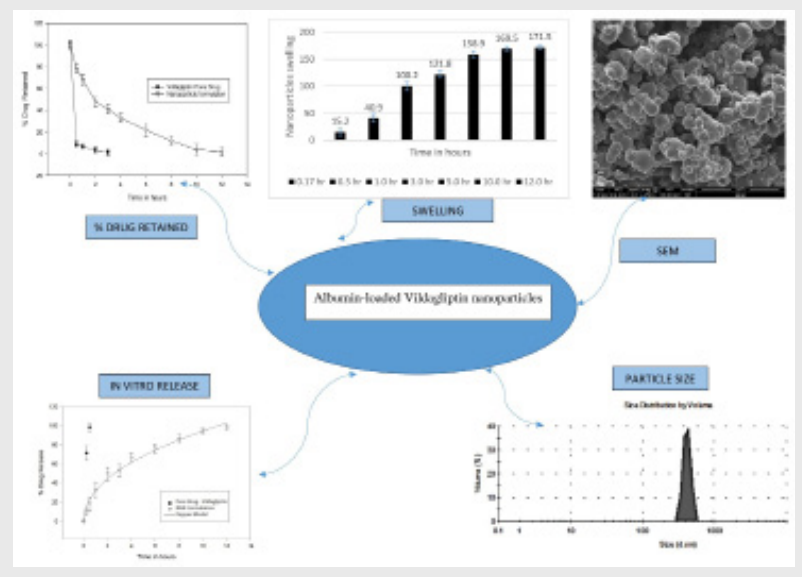

\section{SUMMARY}

- Spray-drying is a most common interesting techniques for many new drug development studies. Vildagliptin prolonged retention in GIT $(\sim 12 \mathrm{~h})$ further suggests benefit associated with oral delivery of nanoparticles with constant drug delivery.

\section{ABOUT AUTHORS}

Dr. Sree Harsha received his (ranked top 5) Master of Pharmacy Degree and subsequently earned a doctorate in Pharmaceutics from Rajiv Gandhi University of Health Sciences, Bangalore, India in 2006. He came to King Faisal University in 2007 as an assistant professor in the Department of Pharmaceutical Sciences, bringing with him several years' worth of teaching experience in fundamentals of pharmaceutics and drug delivery systems. He was actively participated in Accreditation Council of Pharmacy Education (ACPE) and Canadian Council for the Accreditation of Pharmacy Programs (CCAPP). His primary area of focus is pharmaceutical technology and novel/ targeted drug delivery systems. For this research, he received grants (20 number) from Deanship of Scientific research, King Faisal University. The author contributed so far to 50 peer-reviewed full papers on a variety of topics in lung targeting, topical drug delivery, and mucoadhesive drug delivery systems, He has contributed in writing a book chapter titled "Targeted Drug Delivery System" and "Microspheres" in Textbook of Industrial Pharmacy, Publisher-Orient Longman Private Ltd. In addition, he is an Ad-hoc reviewer for scientific journals. He has attended many seminars and Workshop both national and international on Pharmaceutical Technology and Public health issues.

Cite this article: Nagaraja SH, Al-Dhubiab BE, Tekade RK, Venugopala KN, Ghorpade RV, Meravanige G, Alqadheeb A. Novel Preparation and Effective Delivery of Mucoadeshive Nanoparticles Containing Anti-diabetic Drug. Indian J of Pharmaceutical Education and Research. 2019;53(2S):s43-s49. 